\title{
Rate of Molecular Transfer of Allyl Alcohol across an AOT surfactant layer using
}

\section{Muon Spin Spectroscopy}

\author{
Upali A. Jayasooriya ${ }^{1}$, Nigel J. Clayden ${ }^{* 1}$, David C. Steytler ${ }^{1}$, Vasily S. Oganesyan, Jamie N.T. \\ Peck$^{1}$, Rustem Khasanov ${ }^{2}$, Robert Scheuermann², and Alexey Stoykov². \\ 'School of Chemistry, University of East Anglia, Norwich, NR4 7TJ, UK \\ ${ }^{2}$ Laboratory for Muon-Spin Spectroscopy, Paul Scherrer Institut, WBBA/120, CH-5232 Villigen PSI, Switzerland
}

KEYWORDS soft matter, muon spin spectroscopy, microemulsion, chemical exchange,

\begin{abstract}
The transfer rate of a probe molecule across the interfacial layer of a water-in-oil (w/o) microemulsion was investigated using a combination of Transverse Field muon spin rotation (TF- $\mu$ SR), Avoided Level Crossing muon spin resonance (ALC- $\mu S R)$ and Monte Carlo simulations. Reverse microemulsions consist of nanometer sized water droplets dispersed in an apolar solvent separated by a surfactant monolayer. Although the thermodynamic, static model of these systems has been well described, our understanding of their dynamics is currently incomplete. For example, what is the rate of solute transfer between the aqueous and apolar solvents, and how this is influenced by the structure of the interface? With an appropriate choice of system and probe molecule, $\mu$ SR offers a unique opportunity to directly probe these interfacial transfer dynamics. Here, we have employed a well characterized w/o microemulsion stabilized by bis(2-ethylhexyl) sodium sulphosuccinate (Aerosol OT), with allyl alcohol $\left(\mathrm{CH}_{2}=\mathrm{CH}-\mathrm{CH} 2-\mathrm{OH}, \mathrm{AA}\right)$ as the probe. Resonances due to both muoniated radicals, $\mathrm{CMuH}_{2}-\mathrm{C}^{*} \mathrm{H}-\mathrm{CH}_{2}-\mathrm{OH}$ and $\mathrm{C}^{*} \mathrm{H}_{2}-\mathrm{CHMu}-\mathrm{CH}_{2}-\mathrm{OH}$, were observed with the former being the dominant species. All resonances displayed solvent dependence, with those in the microemulsion observed as a single resonance located at intermediate magnetic fields to those present in either of the pure solvents. Observation of a single resonance is strong evidence for interfacial transfer being in the fast exchange limit. Monte-Carlo calculations of the $\Delta \mathrm{M}=\mathrm{o}$ ALC resonances are consistent with the experimental data, indicating exchange rates greater than $10^{9} \mathrm{~s}^{-1}$, placing the rate of interfacial transfer at the diffusion limit.
\end{abstract}

\section{Introduction}

Water-in-oil (w/o) microemulsions consist of nanometre-sized, spherical water droplets dispersed in an apolar solvent with a surfactant monolayer preventing unfavourable oil-water contact. ${ }^{1}$ Interest in microemulsions stems from their importance in a range of applications including biocatalysis, ${ }^{2}$ nanoparticle synthesis ${ }^{3}$ and membrane-mimetic studies. 4 One of the most commonly studied w/o microemulsions is the ternary system comprising bis(2ethylhexyl) sodium sulphosuccinate (AOT), water and hydrocarbon for which the water droplet size $\left(R_{w}\right)$ can be easily controlled through the water-to-surfactant molar ratio $(W)$. In simple terms a microemulsion can be thought as containing two solvent domains, one inside the micelle and one outside. More sophisticated approaches describe regions in proximity to the interface where bound water or solvent penetration into the surfactant layer may occur. Indeed for AOT, a variety of techniques have shown that when the micelle is small, up to approximately, $W=10$, the water contained shows restricted dynamics. However, with larger micelles, $W=20$ such as used in the current work, the water dynamics is essentially identical to that of bulk water.5,6 Even less is known about the effect of the surfactant tails ordering the oil.

Although there is an extensive literature describing the thermodynamic, static picture, our understanding of the dynamic aspects is not so complete. ${ }^{7}$ Yet microemulsions are an intrinsically dynamic system ${ }^{8}$ with a range of processes taking place: internal motions of components, droplet diffusion and exchange of contents of water droplets and surfactant during sufficiently energetic droplet collisions. One question which has been little investigated is the rate of molecular transfer across the interface separating the water and oil domains, and how this is influenced by the structure of the surfactant layer. Many of the probe molecules used in dynamic studies locate preferentially with the interface and for this reason more emphasis has been placed on examining the interfacial region itself. An example being when fluorescence quenching is used with non-polar quencher and polar excited probes and vice versa.9,10 When the solute is insoluble in the oil phase, alternative mechanisms for the transfer of solute between micelles are required, one such being transient droplet coalescence followed by separation..$^{11,12}$ 
An alternative way of measuring the rate of solute exchange is to use ESR and spin probes. ${ }^{13,14}$ The essential idea being that the spin probe shows different ESR parameters, specifically hyperfine interactions, in water, interface regions and hydrocarbon as solvent. Exchange of the spin probe between these environments leads to the averaging of the ESR parameters, in a process known as "chemical exchange". ${ }^{15}$ By simulating the spectral changes it is possible to derive the exchange rate between the environments. Thus in the case of the spin probe 2,2,6,6,-tetramethyl-4piperidone $\mathrm{N}$-oxide in an AOT w/o microemulsion in heptane a rate of exchange of $4 \times 10^{6} \mathrm{~s}^{-1}$ was deduced. However, the exchange observed was interpreted as being between a water pool and interface region with possible exchange with the external heptane being ignored. The principal limitation of this method is the restricted class of spin probes.

An alternative method with possibly wider applicability is to create a radical by implanting a muon ${ }^{16-19}$ into a reverse micelle system containing an appropriate precursor molecule. An excellent introduction to the technique as well as a schematic description of the different muon experiments, Figures 2 and 4 can be found in reference 17 . Positively charged muons $\left(\mu^{+}\right)$can be implanted into the microemulsion system, using a surface muon beam which is nearly $100 \%$ spin polarized, with an approximately circular spot of roughly $1 \mathrm{~cm}$ full width at half maximum at the sample position. After losing energy the muon comes to rest within a $\mathrm{mm}$ or so of the surface of the material, frequently thermalizing by gaining an electron to give muonium which may then react with centers of unsaturation (double or triple bonds), to form a paramagnetic species known as a muoniated radical, a magnetic probe. Like ESR the muoniated radical will be sensitive to the solvent through different hyperfine coupling constants and the rate of transfer will be studied through simulation of the muon spectral properties. ${ }^{19}$ Consequently, with a judicious choice of system and "precursor molecules", $\mu$ SR offers a distinctive opportunity to probe directly interfacial transfer dynamics and energetics. One advantage over the ESR method is the possibility of using a variety of smaller probe molecules, ones less likely to interact with the surfactant layer.

Although muons are a short lived subatomic particle and as such any muoniated radicals will decay with a lifetime of $2.2 \mu \mathrm{s}$ it is important to appreciate that the muoniated radical species are being constantly created in the sample by muon implantation at a rate of $10^{7}$ muons $\mathrm{s}^{-1}$. The stability of muoniated radicals is unaffected by the general experimental conditions though the presence of oxygen can lead to increased relaxation rates to the extent that deoxygenation of the solutions is preferred. Given the muon will tend to add indiscriminately to unsaturated organic compounds there is also the possibility of additional radical products being formed in a chemically complex system. However, the dependence of the hyperfine coupling con- stants on the chemical environment often results in discrimination in the resulting ALC- $\mu$ SR spectrum. Thus muon spin spectroscopy can in principle be used to study the competitive transfer of solutes across an interface.

Muon spin resonance has previously been used to study surfactant systems, and in particular co-surfactant partitioning between the surfactant and an aqueous phase. Here a solvent polarity scale was constructed based on the solvent dependence of the hyperfine coupling constants. ${ }^{21}$, ${ }^{22}$ Importantly, it was observed that the solvent polarity associated with the co-surfactant was $55 \%$ which is consistent with rapid exchange between an aqueous environment (100\% polar) and a more hydrophobic one, or the muon being localized in a region of intermediate polarity, perhaps by the surfactant head group. Notably, the authors concluded that the rate of diffusion in their system was consistent with the observation of an exchange process but interpreted their data in terms of a localized intermediate environment in the light of spin exchange data. ${ }^{23}$ Spin exchange being a phenomenon whereby the relaxation of the muon is enhanced by a local paramagnetic ion. The location of this ion can in turn be restricted by its solubility in a particular phase hence allowing the solvent accessibility to the muoniated radical to be determined.

Avoided Level Crossing muon spin resonance (ALC$\mu \mathrm{SR})^{18}$ can be used to determine the nuclear hyperfine coupling constants. In this technique the longitudinally applied magnetic field is varied until resonances are seen corresponding to the avoided level crossings in the three spin system (muon, proton and electron) as determined by the magnitude of the muon and nuclear hyperfine coupling constants. In the solution state only the $\Delta \mathrm{M}=\mathrm{o}$ transitions (muon-nuclear spin flip-flop) are observable. The muonelectron hyperfine coupling constants are determined independently in a transverse field $\mu S R$ experiment where resonances due to the $\Delta \mathrm{M}= \pm 1$ transitions are observed. ${ }^{18}$

To provide a large interfacial area required for the experiments, a $W=20 \mathrm{w} / \mathrm{o}$ microemulsion stabilized by AOT was employed with $\mathrm{n}$-heptane as the oil phase. This system provides small water droplets $\left(R_{w} \sim 3.6 \mathrm{~nm}\right)$ that exhibit a fairly wide thermal stability window $\left(\mathrm{o}-50^{\circ} \mathrm{C}\right)$ suitable for temperature variation studies. The precursor molecule chosen was allyl alcohol $\left(\mathrm{CH}_{2}=\mathrm{CH}-\mathrm{CH}_{2}-\mathrm{OH}, \mathrm{AA}\right)$. Here the unsaturated center fulfils the requirements for muon capture while the size and polarity ensure the balanced partitioning between the water droplets and surrounding oil phase that is essential in the proof of principle measurements. Moreover, allyl alcohol can be added to the microemulsion system at the levels required for effective muon capture particularly for TF experiments $\left(1 \mathrm{~mol} \mathrm{dm}^{-3}\right)$, without destroying the droplet structure.

\section{Experimental Section}

Sample Preparation

AOT (Sigma-Aldrich) was purified by contacting a solution of the surfactant in diethyl ether with activated charcoal $(\mathrm{BDH})$, followed by filtration through a $0.2 \mu \mathrm{m}$ filter 
and solvent evaporation in vacuo. This procedure has previously been found effective in removing polar impurities, such as succinic acid and 2-ethylhexanol. ${ }^{23}$ Allyl alcohol (99\%), and n-heptane (Chromasolv grade) were obtained from Sigma-Aldrich and de-ionised water was used throughout. Allyl alcohol containing microemulsions were prepared in heptane with concentrations of $0.5 \mathrm{M}$ AOT, 10 $\mathrm{M}$ water and 0.07 volume fraction of allyl alcohol. Solutions of allyl alcohol in heptane and water for comparative muon experiments were prepared at the same concentration. All samples were transferred into glass bulbs (diameter $=3.5$ $\mathrm{cm}$ ), subjected to 10 cycles of freeze-pump-thaw to remove any dissolved oxygen. For transverse field measurements (TF- $\mu \mathrm{SR}$ ), on the GPD instrument at the Paul Scherrer Institute, the bulbs could be directly loaded into the cryostat. For ALC measurements samples were transferred from the glass bulbs into the metal liquid cells at PSI under an oxygen free helium atmosphere.

Avoided Level Crossing Muon Spin Resonance Spectroscopy (ALC- $\mu \mathrm{SR})$

ALC- $\mu S R$ is a technique widely used to determine nuclear hyperfine coupling constants. A full description of the background theory and further references can be found in a number of reviews. ${ }^{17,18}$ The key feature to appreciate is that the muon, proton and electron spins interact through muon and nuclear hyperfine couplings. Together these combined states show an energy dependence on the applied magnetic field. At certain values of the applied magnetic field, the magnetic energy levels that would otherwise cross, mix and repel one another. This mixing of levels gives rise to a depolarization of the muon spin, the detection of which provides a way of sensing these crossings, as a resonance in the ALC- $\mu$ SR spectrum. The positions of these resonances are determined by the muon and nuclear hyperfine coupling constants which in turn reflect the local electron densities at these nuclei. Three types of resonance are possible, those with no overall change in the muon, nuclear and electron spin, $\Delta \mathrm{M}=\mathrm{o},\left(\Delta_{\mathrm{o}}\right)$ those with a net change of $\Delta \mathrm{M}= \pm 1,\left(\Delta_{1}\right)$ and those where $\Delta \mathrm{M}= \pm 2,\left(\Delta_{2}\right)$. Only the $\Delta_{\mathrm{o}}$ resonances are relevant to this work because of the rapid isotropic motion of the molecules in the liquid state.

ALC- $\mu$ SR experiments were performed on the spectrometer at beamline $\pi \mathrm{E}_{3}$ of the Swiss Muon Source, "S $\mu S$ ", Paul Scherrer Institut, Villigen, Switzerland. Resonance spectra (i.e., the field dependence of the time-integrated muon spin polarization) were recorded over the field ranges appropriate for all the resonances, typically $1.2-2.2 \mathrm{~T}$. Measurements were made over the temperature range 275-330 $\mathrm{K}$. Additional ALC- $\mu \mathrm{SR}$ experiments were carried out on the HiFi spectrometer at ISIS, Rutherford Appleton Laboratory, United Kingdom.

In order to derive the nuclear hyperfine coupling constants, the muon hyperfine coupling constants must first be determined from TF- $\mu S R$, experiments. These trans- verse field muon spin rotation spectra were measured using the GPD spectrometer at PSI (area $\mu \mathrm{EI})$ with a transverse magnetic field of $0.3 \mathrm{~T}$ at the sample position. Muon coupling constants of the muoniated allyl alcohol in the water, heptane and microemulsion systems were each determined from the analysis of the precession signals. Each muoniated radical species present gives rise to a pair of resonances $\left(\Delta_{1}\right)$, the frequencies of which are split by the muon hyperfine coupling constant. The $\Delta_{\mathrm{o}}$ resonance positions observed in the ALC- $\mu$ SR spectra were then used together with the muon hyperfine coupling constants to calculate the nuclear hyperfine coupling constants using Equation 1: ${ }^{24}$

$$
B_{r}\left(\Delta_{0}\right)=\frac{A_{\mu}-A_{p}}{2\left(\gamma_{\mu}-\gamma_{p}\right)}-\frac{A_{\mu}+A_{p}}{2 \gamma_{e}} \quad 1
$$

Where $A_{\mu}$ and $A_{p}$ denote the muon and nuclear hyperfine coupling constants respectively and $\gamma_{\mu}, \gamma_{p}$ and $\gamma_{e}$ denote the gyromagnetic ratios of the muon, proton and the electron, respectively.

\section{Spectral simulations}

A primary or secondary radical may be formed when muons add to allyl alcohol to form the muoniated species shown in, Figure 1. In order to assist in the understanding of the observed ALC- $\mu$ SR and transverse muon spin rotation spectra, the hyperfine parameters of these radicals were estimated using ab initio density functional calculations.

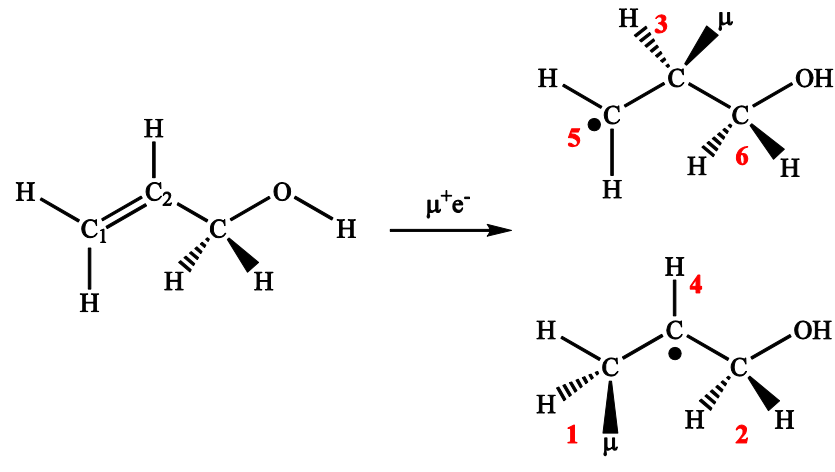

Figure 1. Muonium addition to the double bond in allyl alcohol, $\mathrm{CH}_{2}=\mathrm{CH}-\mathrm{CH}_{2} \mathrm{OH}$, to form the two isomers of the muoniated radical, corresponding to $\mathrm{C}-1$ and $\mathrm{C}-2$ addition of muonium to the double bond. The numbers in the figure are the assignments of the proton groups to the experimentally observed ALC- $\mu$ SR resonances shown in Figure 3.

\section{DFT Computational details.}

DFT calculations were performed on an isolated molecule of allyl alcohol using the Gaussian 03 software package. ${ }^{25}$ All calculations used the Becke3LYP (B 3 LYP) hybrid GGA functional ${ }^{26-28}$ in conjunction with the $6-311+G(d, p)$ 
basis set ${ }^{29}$ for the geometry optimization and energy calculation and EPR-III basis set for the calculation of hyperfine coupling constants. ${ }^{30}$ Structures were confirmed as minima through frequency calculations. Calculations were performed using hydrogen analogue systems. To account for the light mass of the muon relative to the hydrogen, the bond length was scaled by a factor of 1.02.31 The isotropic and dipolar muon and proton hyperfine coupling constants are proportional to each other with the scaling factor $\mathrm{K}$, namely $A_{\mu}=\mathrm{K} \cdot A_{p} \cdot{ }^{32}$ In this paper $\mathrm{K}=4.55$ and 4.65 were employed for terminal and central $\mathrm{Mu}$ attachments respectively which provide the best agreement with the observed ALC spectra and is consistent with the values determined and reported in the literature for the $\mathrm{C}_{6} \mathrm{H}_{6} \mathrm{Mu}$ radicals. ${ }^{32}$

Rotational averaging of the hyperfine coupling values for both $\mathrm{Mu}$ and different $\mathrm{H}$ atoms in each muoniated radical have been performed according to the procedure described previously. ${ }^{33}$ Whilst maintaining a fixed $\mathrm{C}-\mathrm{Mu}$ bond distance, two slices of the potential energy surface were calculated by scanning two dihedral angles adjacent to the carbon atom connected to $\mathrm{Mu}$. At each increment, corresponding $A_{\mu}$ and $A_{\mathrm{p}}$ values for each proton were calculated. All geometries were initially optimized in the gas phase. Solvation was accounted for by single point energy calculations using the integral equation formalism polarizable continuum model (IEFPCM) in either water $(\varepsilon=78.35)$ or heptane $(\varepsilon=1.91)$, in combination with the united atom topological model for radii setting (RADII=UAHF).

Data analysis

Transverse field data were Fourier transformed using the $\mathrm{fftx}$ fitting program from PSI and fitted in the time domain using minfit. ALC data were background subtracted and fitted using the ROOT software package developed at CERN and provided by $\mathrm{H}$. Dilger. Computer simulations of the chemical exchange process in ALC- $\mu \mathrm{SR}$ spectra were carried out by Monte Carlo methods ${ }^{33}$ using the FORTRAN program MofitGS adapted from the original program ALCMo obtained from E. Roduner. Partitioning of the allyl alcohol between the water and heptane was take into account through the probabilities associated with creating the muoniated allyl alcohol in a particular phase and in the exchange step. A flow chart illustrating the calculation of the muon spectrum by the Monte Carlo methods is presented in reference 33. In the current calculation the site exchange subroutine was used. Here, the transfer rate is simply the inverse of the time increment. At each successive time, during the lifetime of the muon, the muon may undergo an exchange between the water and heptane depending on a generated random number. The probability of the muon exchange at each time increment, determined by the range of the random number, will reflect the partitioning of the allyl alcohol between the water and heptane phases. The only fitting parameter was the time increment which corresponds to the lifetime of a muon in a particular environment. In the program the time increment was stepped through a range of values and for each value the
ALC- $\mu$ SR spectrum calculated and compared with the experimental spectrum giving a $\chi^{2}$ value for the fit. Typical experimental errors in the experimental ALC- $\mu$ SR spectra were of the order of $8 \times 10^{-5}$. Owing to the intrinsic random decay of a muon the simulated ALC- $\mu$ SR spectrum showed fluctuations in the polarization corresponding to a noise

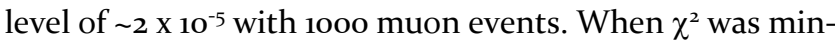
imized, usually of the order of 1200, the transfer rate was taken as the inverse of the time increment at the $\chi^{2}$ minimum.

Although the reduced $\chi^{2}$ value is somewhat high at 1.7, this needs to be seen within the context of fitting to a single parameter, the inverse of the global exchange rate, without allowing fitting of the hyperfine coupling constants. These were taken as those seen for the muoniated allyl alcohol in pure heptane and water. Some optimization in the hyperfine coupling constants was carried out only to ensure the ALC resonance minima occurred at the experimentally observed magnetic fields however to allow these to be fitting parameters would unnecessarily complicate the fitting procedure and add little to the confidence we would have in the fit.

\section{Results and Discussion}

In discussing the results, first the assignment of the experimental TF- $\mu \mathrm{SR}$ and ALC- $\mu \mathrm{SR}$ will be considered along with the evidence for the exchange of allyl alcohol between oil and water phases. Then the use of computer simulations of the lineshapes to determine the rate of this exchange, will be described.

\section{TF- $\mu \mathrm{SR}$}

Initial TF- $\mu \mathrm{SR}$ experiments examined the formation of muoniated radicals in the pure solvents water and heptane. Two muoniated radicals can be formed by the addition of muonium to allyl alcohol, Figure 1, both of which can be expected to give a $\mu \mathrm{SR}$ signal in the TF- $\mu \mathrm{SR}$ spectrum. However, only a single set of resonances were seen in the TF- $\mu$ SR spectrum for allyl alcohol in water and heptane, Figure 2, suggesting only one of the two possible radicals is promptly produced and thus appears in this spectrum. Slowly forming radicals may not appear in TF- $\mu$ SR spectra because the large transverse magnetic fields used cause excessive dephasing of the muon spins and loss of signal intensity. 


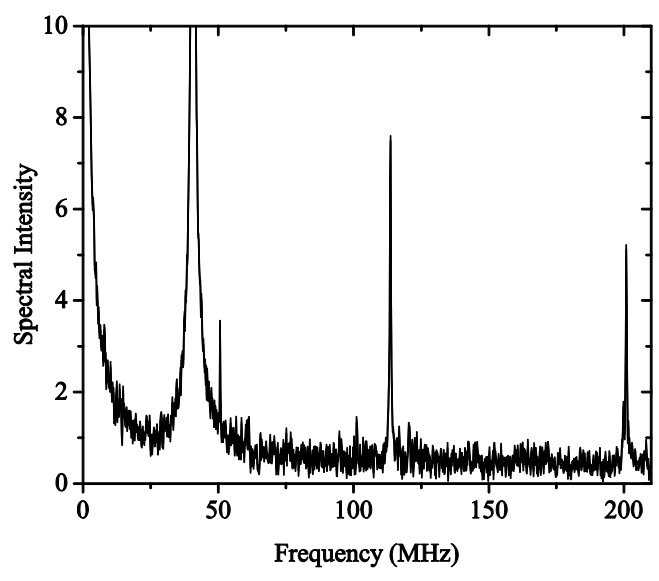

Figure 2. TF- $\mu$ SR spectrum from $0.5 \mathrm{M}$ allyl alcohol in water at $288.5 \mathrm{~K}$ in a transverse magnetic field of $0.3 \mathrm{~T}$. The spectral feature at $40.67 \mathrm{MHz}$ (truncated to view the other signals better) is from muons in a diamagnetic environment. The pair of signals at $113.60 \mathrm{MHz}$ and $200.81 \mathrm{MHz}$ result from one of the two radicals.

Such an observation would be consistent with the preference shown in muon addition across double bonds to give secondary rather than primary radicals. ${ }^{36}$ Reasonable agreement is seen between the calculated and experimental muon hyperfine coupling constants for the muon addition product to the $\mathrm{C}-1$ of the allyl alcohol, leading to the secondary radical, in both water and heptane.

\begin{tabular}{llll}
\hline $\begin{array}{l}\text { Muonium } \\
\text { addition } \\
\text { site }\end{array}$ & Solvent & $\begin{array}{l}\text { Experimental } \\
A_{\mu} / \mathrm{MHz}\end{array}$ & $\begin{array}{l}\text { Calculated } \\
A_{\mu} / \mathrm{MHz}\end{array}$ \\
\hline $\mathrm{C}_{1}$ adduct & water & 314.4 & $323 ; 330$ \\
\hline & heptane & 309.5 & $321 ; 328$ \\
\hline $\mathrm{C}_{2}$ adduct & water & Not observed & $370 ; 378$ \\
\hline & heptane & Not observed & $364 ; 372$ \\
\hline
\end{tabular}

*Two calculated vales are shown corresponding to the two values used for the scaling factor, $\mathrm{K}$

Table 1. Comparison of the muon-electron hyperfine coupling constants, $A \mu$, determined from experimental TF- $\mu S R$ data with the values obtained from DFT calculations.

Turning now to the TF- $\mu$ SR spectrum of the microemulsion, the notable feature is that only a single pair of frequencies was seen for the microemulsion. Allyl alcohol is roughly equally partitioned between water and heptane, thus we might have expected to see two pairs of frequencies for the muoniated allyl alcohol, one from those created in water and another those in the heptane. An accidental coincidence of the frequencies cannot explain this since it is clear from the reference TF- $\mu \mathrm{SR}$ spectra that significantly different muon hyperfine couplings are seen for the muoniated radical in water and heptane. Moreover, the ease with which TF- $\mu$ SR spectra could be obtained for the allyl alcohol in both water and heptane means it is implausible that in the microemulsion muons add only to the allyl alcohol in one of the phases. Yet as noted above only a single pair is seen and significantly are at frequencies corresponding to a muon hyperfine coupling constant representing an average of that seen for water and heptane. Given that the allyl alcohol partitions between the solvents and is not localized in some other phase, the presence of only one pair of frequencies is conclusive evidence of an exchange process.

Confirmation that exchange must be taking place within the lifetime of the muon was provided by the observation of broadening of the resonances in the TF- $\mu$ SR spectra when a paramagnetic ion, $\mathrm{Ni}^{2+}$, was added to the aqueous phase. Paramagnetic ions can cause linebroadening through either a dipole-dipole mechanism or spin exchange mechanism resulting from collisions. ${ }^{16}$ Regardless of the mechanism, the paramagnetic ion and the species being relaxed must be close, within a nm or less. However, the paramagnetic ion chosen, $\mathrm{Ni}^{2+}(\mathrm{aq})$, was only soluble in the aqueous phase consequently to see a Gaussian lineshape represented by a single linewidth implies any muoniated allyl alcohol being formed in the heptane must be able to transfer rapidly through into the water core of the droplets. Overall the relaxation rate $\lambda_{\mu}$ can be expressed as Equation 2:

$$
\lambda_{\mu}=\lambda_{0}+R\left[\mathrm{Ni}^{2+}\right]
$$

Where $\lambda_{0}$ is the intrinsic relaxation rate and $R$ the relaxivity of the paramagnetic ion.

\begin{tabular}{llll}
\hline & $\lambda_{\mu} / \mu \mathrm{s}^{-1}$ & $\lambda_{\mathrm{o}} / \mu \mathrm{s}^{-1}$ & $R\left[\mathrm{Ni}^{2+}\right] / \mu \mathrm{s}^{-1}$ \\
\hline Water & 7.04 & 0.77 & 6.27 \\
\hline Microemulsion & 3.77 & 1.68 & 2.09 \\
\hline
\end{tabular}

Table 2. Effect of added $\mathrm{Ni}^{2+}$ on the TF- $\mu \mathrm{SR}$ relaxation rates at $299 \mathrm{~K}$

If the relaxivity in the water core of the microemulsion droplet is assumed to be the same as for bulk water then the reduced relaxation rate seen for the microemulsion must reflect the partitioning of the radical between the water and heptane. A rough estimate then would be a value of $2.09 / 6.27=0.33$ for the partition coefficient, close to the accepted value of 0.4 . Thus the enhanced relaxation caused by the addition of $\mathrm{Ni}^{2+}$ is consistent with exchange of the radical across the surfactant layer at a rate which is fast on the hyperfine constant timescale.

All the muon hyperfine coupling constants showed a linear and similar temperature dependence as set out in Table 3.

\begin{tabular}{llll}
\hline & Water & Heptane & Microemulsion \\
\hline$A_{\mu}(300 \mathrm{~K})$ & 313.4 & 309.5 & 311.2 \\
\hline
\end{tabular}




\begin{tabular}{llll}
\hline $\mathrm{MHz}$ & & & \\
\hline $\mathrm{d} A_{\mu} / \mathrm{dT}$ & -0.1934 & -0.1834 & -0.1882 \\
$\mathrm{MHz} / \mathrm{K}$ & & & \\
\hline
\end{tabular}

Table 3. Linear Temperature Dependence of the Muon Hyperfine Coupling Constants for the terminal addition allyl alcohol radical

\section{ALC- $\mu S R$}

ALC- $\mu$ SR spectra of allyl alcohol in water, $\mathrm{n}$-heptane and in the micro-emulsion are shown in Figure 3. Five resonances were observed and are numbered 1 to 5 in ascending field values. A resonance is, in principle, expected for every hydrogen in the muoniated radical at which there is unpaired electron density. These resonances, because of the isotropic motion of the radical in solution, can be assigned to the $\Delta \mathrm{M}=\mathrm{o}$, or flip-flop transitions of the muon spin with the spins of protons in allyl alcohol. Using the DFT estimates for the applied field resonance values, $\mathrm{B}_{\text {calc }}$, it is possible to obtain an unambiguous assignment for these resonances as shown in Table 4

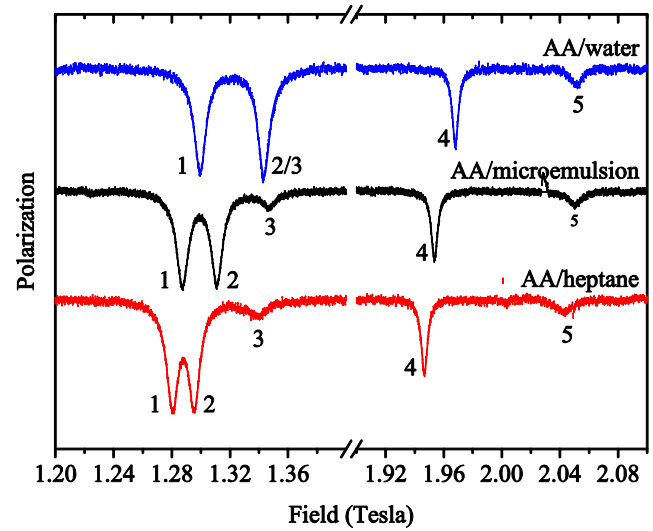

Figure 3. ALC- $\mu \mathrm{SR}$ resonances observed for the allyl alcohol solutions in water, micro-emulsion and n-heptane at $330 \mathrm{~K}$. The resonances are numbered 1 to 5 in ascending order of the field.

\begin{tabular}{llllll}
\hline Medium & $\begin{array}{llll}\mathrm{H} \\
\text { water }\end{array}$ & $\begin{array}{l}\mathrm{A}_{\mu} \text { Calc } \\
/ \mathrm{MHz}\end{array}$ & $\begin{array}{l}\mathrm{A}_{\mathrm{H}} \text { Calc } \\
/ \mathrm{MHz}\end{array}$ & $\begin{array}{l}\mathrm{B}_{\text {res }} \\
\text { Calc/ } \\
\mathrm{T}\end{array}$ & $\begin{array}{l}\mathrm{B}_{\text {res }} \\
\text { Expt/ } \\
\mathrm{T}\end{array}$ \\
\cline { 2 - 6 } & 1 & 322.8 & 72.40 & 1.34 & 1.34 \\
\cline { 2 - 6 } & 2 & 329.9 & 73.22 & 1.37 & 1.39 \\
\cline { 2 - 6 } & 3 & 369.6 & 101.5 & 1.43 & 1.39 \\
\hline \multirow{2}{*}{ heptane } & 329.9 & -59.92 & 2.09 & 2.09 \\
\cline { 2 - 6 } & 1 & 377.7 & -62.16 & 2.36 & $\mathrm{n} / \mathrm{o}$ \\
\cline { 2 - 6 } & 2 & 369.6 & -0.31 & 1.98 & 2.01 \\
\cline { 2 - 6 } & 3 & 363.7 & 102.8 & 1.40 & 1.38 \\
\cline { 2 - 6 } & 4 & 327.8 & -58.61 & 2.07 & 2.08 \\
\hline
\end{tabular}

\begin{tabular}{lllll}
\hline 5 & 371.7 & -61.99 & 2.33 & n/o \\
\hline 6 & 363.7 & -0.48 & 1.95 & 1.98
\end{tabular}

Table 4. DFT predictions of the resonance fields, and their assignments to the experimentally observed resonances in the ALC- $\mu$ SR spectra. For H positions 1 to 6, see Figure 1.

As remarked earlier two muoniated radicals are expected to be formed by the addition of muonium to allyl alcohol, Figure 1, both are clearly discernible in the ALC- $\mu$ SR spectra, Figure 3, as confirmed by the DFT predictions. This is in contrast to the TF- $\mu \mathrm{SR}$ spectra. A possible explanation for this difference lies in the differing rates of formation of the two radicals since, as noted earlier, in order for a radical to be detected in the TF- $\mu$ SR spectrum, it must be formed within a few nanoseconds of muon implantation. Otherwise the greater transverse magnetic field will cause excessive dephasing of the muon spins and loss of signal intensity. Consequently, slowly formed radicals are not accessible to the TF- $\mu S R$ experiment. Thus the primary radical is absent in the TF- $\mu$ SR spectrum because it forms more slowly and the muon spins are more quickly dephased due to precession about a transverse magnetic field compared to the longitudinal field of the ALC- $\mu$ SR configuration. Further, such an observation would be consistent with the general observation of the preference shown in muonium addition reactions across double bonds to give preferentially secondary rather than primary radicals. ${ }^{34}$ It is of note that there is reasonable agreement (Table 4) between the calculated and experimental muon-electron hyperfine coupling constant for the muonium addition product to the $C_{1}$ of the allyl alcohol, leading to the secondary radical, in both water and heptane.

All resonances both in the ALC- $\mu \mathrm{SR}$ and TF- $\mu \mathrm{SR}$, were solvent dependent, reflecting the different polarities of water and heptane. In the case of the TF- $\mu$ SR spectra, the presence of a single pair of frequencies is evidence for exchange between the water and heptane phases. Further evidence for an exchange process is provided by the single set of resonances seen in the ALC- $\mu$ SR spectra for the muoniated allyl alcohol radical in the microemulsion. The fact a single set of resonances are seen, lying in between those seen for the equivalent resonances in the pure solvents, strongly suggests that this exchange process must be on the fast limit side of the coalescence point. Critically, there was no evidence for muoniated radicals existing solely in one or other of the phases. This must therefore correspond to rapid exchange between different environments because the allyl alcohol partitions almost equally across the hydrocarbon and aqueous phases in this reverse micelle system.

These microemulsions can be considered to consist of three domains, water droplets, surfactant layers and the oil (n-heptane). Each will be characterized by a set of hyperfine coupling constants. In principle, a muoniated radical can diffuse between these three regions and undergo chemical exchange averaging of the hyperfine interactions. 
Absence of discrete ALC resonances corresponding to the three domains is clear evidence of an exchange process. Unfortunately the reverse micellar phase of this microemulsion is not stable at temperatures low enough to observe the slow exchange limit where broadened resonances would be seen at the field values typical of water and heptane. The key question though is whether transfer across the surfactant layer is indeed being observed. At face value this may seem an odd question because allyl alcohol is known to partition between the oil and water, thus exchange must take place, the only question remaining is the rate. Moreover we do not see two resonances typical of a muoniated radical in water or separately in the heptane, consequently whatever exchange is taking place must be on the side of the fast limit. What is being asked is: Could the line broadening reflect the finite time taken to transit the surfactant layer? During this time the muon may adopt the slower rotational dynamics of the microemulsion droplet leading to faster relaxation, rather than because of the exchange process itself. Consequently, in order to assign any exchange broadening, $\lambda_{\mathrm{ex}}$, of the resonance to the transfer process itself it is necessary to rule out any changes to the muon relaxation rate being caused by the radical becoming bound to the reverse micelle and thus showing the global rotational dynamics of the reverse micelle through the $\lambda_{\text {bound }}$ term in the overall relaxation rate:

$$
\lambda=\frac{[A O T: M u A A]}{[M u A A]} \lambda_{\text {bound }}+\left(1-\frac{[A O T: M u A A]}{[M u A A]}\right) \lambda_{\text {free }}+\lambda_{e x}
$$

NMR shows restricted segmental mobility in the AOT 35 and it is plausible that a small molecule could be trapped or bound to the AOT layer. Similarly the muoniated radical could preferentially reside at the interface between the surfactant and bulk solvents, for example through hydrogen bonding at the water/head group interface. Even in these circumstances chemical exchange must still operate, merely that the exchange would be so fast the exchange would not be the cause of the line broadening. Importantly it is not necessary to distinguish between the two and three step processes: [M = micelle $]$

$$
\begin{aligned}
\mathrm{MuAA}_{\text {heptane }}+\mathrm{M} & \underset{\mathrm{k}_{1}}{\rightleftharpoons} \mathrm{MuAA}_{\mathrm{AOT}}: \mathrm{M} \rightleftharpoons \mathrm{MuAA}_{\text {water }}: \mathrm{M} \\
\mathrm{MuAA}_{\text {heptane }}+\mathrm{M} & \underset{\mathrm{k}_{-1}}{\rightleftharpoons} \mathrm{MuAA}_{\text {water }}: \mathrm{M}
\end{aligned}
$$

because if the rate limiting step is the formation of the AOT bound muoniated allyl alcohol (AOT:AA) then this will define the overall rate of transfer across the AOT layer. What is important, however, is establishing whether the $\mathrm{MuAA}_{\mathrm{AOT}}: \mathrm{M}$ bound state can induce significant additional relaxation because of its slower rotational diffusion. Thus we need to estimate $\lambda_{\text {bound. }}$. Using the model for spin relaxation in muoniated radicals developed by Cox and Sivia 37,38 and following the assumptions of McKenzie ${ }^{40}$, relaxation by modulation of the dipolar hyperfine coupling constant by molecular reorientation; fast limit motion because of the absence of $\Delta_{1}$ resonances making the dominant relaxation mechanism $W_{12}$ it is possible to derive the relaxation rate $\lambda$ from

$$
\frac{1}{\tau}=\frac{M_{i j}^{2}}{\lambda}
$$

where $\tau$ is the rotational correlation time, and $M_{\mathrm{ij}}$ is the matrix element leading to relaxation. Assuming further that when bound, the hyperfine coupling constant is averaged by rotational diffusion of the reverse micelle to the same extent as when free it can shown

$$
\lambda_{\text {bound }}=\lambda_{\text {free }}\left(\frac{\tau_{\text {bound }}}{\tau_{\text {free }}}\right)
$$

Representing the reverse micelle and molecule as rotating spheres with a hydrodynamic radius of $r_{\text {bound }}$ and $r_{\text {free }}$ respectively together with Stoke's equation

$$
\tau_{c}=\frac{4 \pi \eta r^{3}}{3 k T}
$$

leads to

$$
\lambda_{\text {bound }}=\lambda_{\text {free }}\left(\frac{r_{\text {bound }}}{r_{\text {free }}}\right)^{3}
$$

Since $r_{\text {bound }} \gg>r_{\text {free }}$ the bound relaxation rate is always going to be greater than the free relaxation rate. Given that the actual concentration of the bound muoniated radical is unknown it will always be possible to model any given additional line broadening on the basis of a three step process. Thus at first sight the $\mu$ SR data will not be able to give a transfer rate. However, this ignores that in the current ALC- $\mu$ SR spectra a number of resonances are seen which would all be averaged by the same chemical exchange rate and yet reflecting a range of differences in hyperfine coupling constants between the solvents. Thus if chemical exchange dominates then these resonances can be expected to show a line broadening dependent on the difference in hyperfine coupling constants with those having a greater variation in hyperfine coupling constants between water and heptane showing greater line broadening in the fast limit. Alterantively, if line broadening was reflecting the change in rotational dynamics of the radical, we would expect a similar line broadening, independent of the difference in hyperfine coupling constants. The marked solvent sensitivity of the ALC resonances is therefore crucial to the study of the rate of interfacial transfer by providing the difference in the magnetic fields required for the ALC resonances in the water and heptane. Here differential line broadening is observed supporting the idea that the line broadening arises from the chemical exchange process itself.

Further evidence that a chemical exchange process was being observed is evident from the temperature depend- 
ence of the resonance linewidths. Only a small temperature dependence was seen for the peaks in the pure solvents, showing that very little change occurs in the relaxation rates of the MuAA radical, $\lambda_{\text {free }}$, as a consequence of changes in the intrinsic rotational dynamics of the radical with temperature. In contrast, marked increases in linewidth occur for the resonances in the microemulsion on cooling. Moreover, the extent of the line broadening was directly related to the magnetic field difference for the ALC resonances in the pure solvents, with more line broadening seen for resonance 2 and less for resonance 1, Figure 4. This dependence is exactly as expected for a chemical exchange process in magnetic resonance, and inconsistent with the idea of an increase in the concentration of a bound $\mathrm{Mu}-$ $\mathrm{AA}_{\mathrm{AOT}}: \mathrm{M}$ complex.
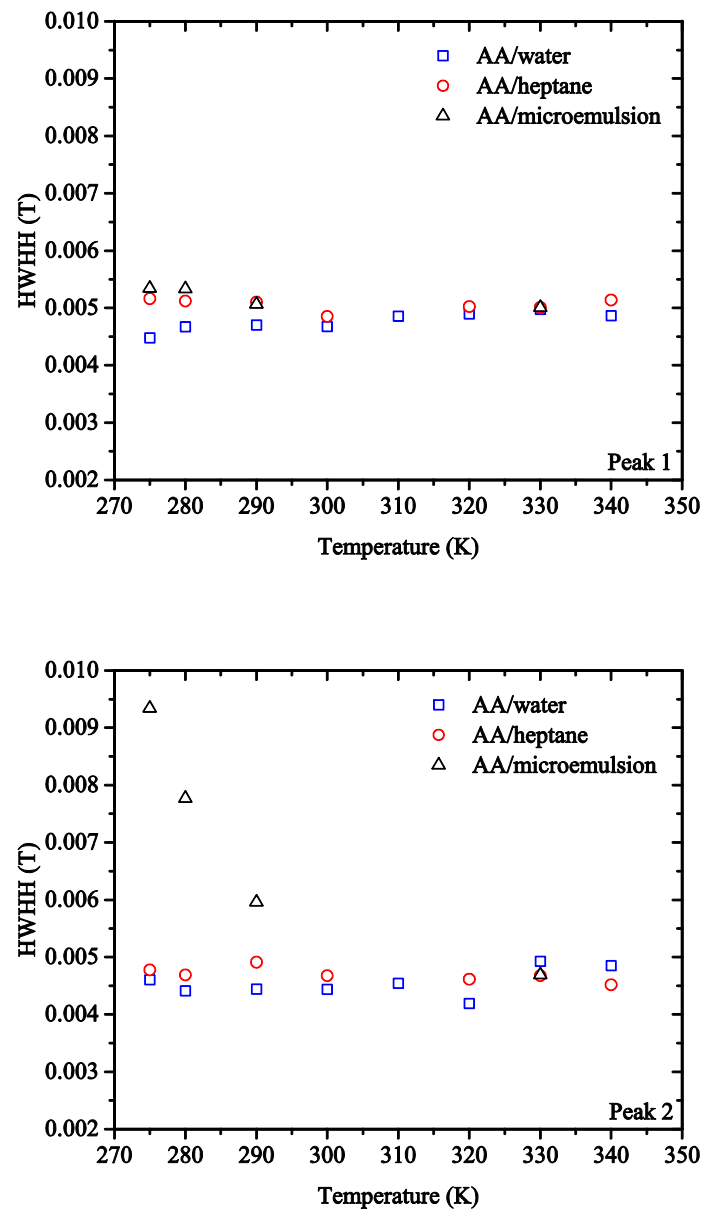

Figure 4: Temperature dependence of the linewidth (HWHH) of the ALC - $\mu$ SR resonances 1 and 2.

Pure water and heptane were used initially as the references for the aqueous and hydrocarbon phases for convenience. In fact, the sodium ion concentration in the aqueous phase is close to $2.8 \mathrm{M}$ while the high concentration of
AOT means that a muon in the heptane may well be diffusing in a medium rich in the hydrocarbon tail groups of the surfactant. However, since SANS measurements indicate that the alkane solvent does not significantly penetrate the AOT alkyl tail region the appropriate reference for the heptane phase is less clear."

ALC- $\mu$ SR spectra were therefore obtained for muoniated allyl alcohol in an aqueous phase, $4 \mathrm{M}$ in sodium ions, and a reverse micellar solution of AOT in heptane. No significant changes were found in the hyperfine coupling constants of resonances 2 and 4 for the radical in the salt solution. While for resonance 1 a shift of only $0.005 \mathrm{~T}$, equal to the magnetic field increment, is seen in the applied magnetic field. This corresponds to $1 \mathrm{MHz}$ in the proton hyperfine coupling constant. Similarly, only small changes in the resonance positions of less than $0.01 \mathrm{~T}$ were seen for the muoniated allyl alcohol radical in a solution of AOT in heptane. Consequently, we can be confident that the conclusion about the presence of fast exchange is correct and any derived rate constants will only be marginally altered.

The result of a typical Monte-Carlo calculation of the $\Delta \mathrm{M}=\mathrm{o}$ ALC resonances expected in the ALC spectra, using a model of exchange between the water and heptane phases, is shown in Figure 5. Only three of the resonances, 1,2 and 4 , gave adequate signal-to-noise ratios for accurate fitting. A global fit was found by varying the same pseudo first order $\left(k_{1}[M]\right)$ exchange rate constant for all the fitted resonances while optimizing the hydrogen hyperfine coupling constants for the radical in the hydrocarbon phase to ensure the averaged value corresponded to the observed applied magnetic fields seen for the microemulsion resonances. This was because of uncertainty in the precise value expected owing to the contribution made by the surfactant tail environment. Although small differences are seen between the observed and calculated lineshapes of

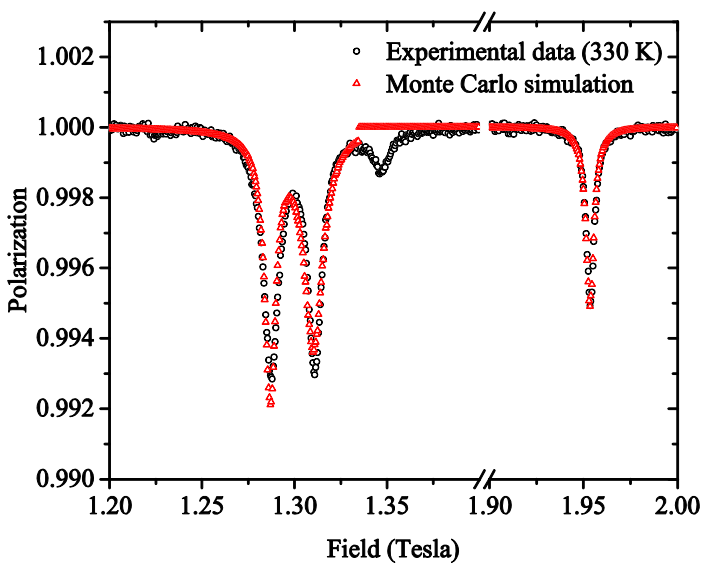

Figure 5. Monte-Carlo simulation33, shown in red, of the ALC$\mu$ SR spectrum, shown in black, at $330 \mathrm{~K}$ based on 5 ,ooo muon events with a $0.5 \mathrm{mT}$ magnetic field increment. Only peaks 1, 2 and 4 are included in the fit. The muon and nuclear hyperfine interactions used in the simulation are given in the table. 
the resonances the overall similarity between the experimental ALC- $\mu$ SR spectra and these simulations indicates that the observed ALC spectra are consistent with the model of a dynamic exchange of the muon between the water and heptane phases across the surfactant layer with exchange rates, $k_{1}[\mathrm{M}]$, ranging from $0.6 \times 10^{8} \mathrm{~s}^{-1}$ at $275 \mathrm{~K}$ to 1.7 $\mathrm{x} 10^{8} \mathrm{~s}^{-1}$ at $330 \mathrm{~K}$. In particular the simulations clearly reveal the differential line-broadening observed for the resonances 1, 2 and 4. Although the errors estimated from the variation in $\chi^{2}$ in the fitting process are small, typically \pm $0.03 \times 10^{8} \mathrm{~s}^{-1}$, this underestimates the true uncertainty because of the presence of nearby local minima giving, for example, the possible range $1.56 \times 10^{8} \mathrm{~s}^{-1}$ to $1.7 \times 10^{8} \mathrm{~s}^{-1}$ at 330 $K$. These rates are similar to those which have been measured for the exchange of dialkyl nitroxides between water and sodium dodecyl sulphate micelles by ESR but without the use of a spin label, demonstrating the unique capabilities of the approach adopted, of using muon spin spectroscopy. ${ }^{40}$

The simulations of the ALC- $\mu$ SR spectra allow us to be confident that an exchange process is being observed. However, we cannot immediately identify the rate constant with the rate of interfacial transfer since it is possible diffusion of the muoniated radical to the interface of the droplet is the rate determining step. Considering in turn each direction of the two step process above in Equation 5. In the forward direction, MuAA must diffuse to a droplet. This process is controlled by the diffusion controlled rate constant, kd for a MuAA: droplet encounter which is $\sim 5 \mathrm{x}$ $10^{10} \mathrm{M}^{-1} \mathrm{~s}^{-1}$. Hence implying a collision frequency of droplets with AA of $\sim 1.1 \times 10^{8} \mathrm{~S}^{-1}$, very similar to the observed exchange rate constant. For the reverse direction we have to consider the diffusion time across the diameter of the water droplets, $\sim 3.1 \times 10^{-9} \mathrm{~s}$ which represents the maximum lifetime in the drop and hence a collision frequency of greater than $3 \times 10^{8} \mathrm{~s}^{-1}$. Therefore, the observed ALC- $\mu \mathrm{SR}$ spectra are consistent with a diffusion controlled process. Nevertheless a lower bound of $\sim 1.7 \times 10^{8} \mathrm{~s}^{-1}$ can be placed on the rate of transfer of allyl alcohol across the surfactant layer. Analysis of the temperature dependence yields an approximate value of $13.4 \mathrm{~kJ} \mathrm{~mol}^{-1}$ for the diffusional process.

\section{Conclusion}

ALC- $\mu$ SR has been investigated as a method for determining the rate of transfer across an AOT surfactant layer which forms the interface between an aqueous and hydrocarbon phase. A single set of ALC resonances were seen for the muoniated radical formed from allyl alcohol consistent with fast exchange. Support for the interpretation in terms of transfer across the AOT layer, rather than the radical lying in an environment of intermediate polarity, was provided by qualitative spin exchange experiments using $\mathrm{Ni}^{2+}$ as well as the known ability of allyl alcohol to partition roughly equally between a water and heptane phase. Moreover the resonances which showed the greatest difference in hydrogen hyperfine coupling constant between water and heptane showed the greatest increases in linewidth with temperature. Monte Carlo simulations of the ALC$\mu \mathrm{SR}$ resonances allowed pseudo first order exchange rate constants of the order of $10^{8} \mathrm{~s}^{-1}$ to be found. Diffusion control of the interfacial transfer appears to be present because the forward diffusion rate is similar to the rate derived from the simulations. A lower bound of $\sim 1.7 \times 10^{8} \mathrm{~s}^{-1}$ can thus be placed on the rate of transfer of muoniated allyl alcohol across the surfactant layer. Back diffusion is estimated to be faster than the observed exchange rate. Despite diffusion control being observed in this system the underlying principle has been clearly established, namely that ALC- $\mu S R$ can be used to monitor fast exchange processes in surfactant systems. When attempting to compare the results obtained by muon spin spectroscopy with existing methods one is struck by the paucity of data and the lack of a systematic study of the transfer process suggesting the measurements are not straightforward to make with the techniques currently available. Classical techniques of concentration jump ${ }^{41}$ and pressure jump ${ }^{42}$ have yielded values of $10^{6}-10^{7} \mathrm{M}^{-1} \mathrm{~s}^{-1}$ for the bimolecular transfer process involving picric acid with AOT and 7,7,8,8-Tetracyanoquinodimethane with dodecylpyridinium respectively. However, quite complex kinetic schemes and experimental methods have limited the scope of these measurements.

In contrast there is an extensive literature using the rotating diffusion cell ${ }^{43,44}$ for determining interfacial transfer rates. Notably it has been used to examine rates of transfer of charged water-soluble probes between microemulsion droplets and a coexisting aqueous phase (Winsor II systems $)^{45}$. However the technique often presents difficulty when applied to the process as reported in this paper, i.e. molecular transfer between oil and water phases through an adsorbed surfactant layer as found in microemulsions. This is due to the low interfacial tension and inevitable formation of microemulsion droplets under the shear conditions imposed by the rotating interface.

Spectroscopic methods based on chemical exchange, like the classical techniques yield homogeneous type rate constants. Despite the possibility of using ESR to measure interfacial exchange rates, to the best of our knowledge, there has not been a single study on microemulsions. One study ${ }^{13,14}$ interpreted the changes in the ESR spectrum in terms of exchange between water pools within the droplet while another looked at the entry rate into SDS micelles where a value of $1.3 \times 10^{10} \mathrm{~mol}^{-1} \mathrm{~s}^{-1}$ was found, close to the diffusion limit ${ }^{40}$. The overwhelming body of ESR work has been on the spin probe localisation as well as ordering and dynamics within the surfactant layer. Similarly there appears to be an absence of data on interfacial transfer in microemulsions using fluorescence spectroscopy. In the light of the weaknesses of existing techniques and the general absence of a systematic study of interfacial transfer in microemulsions is it is all the more important that the wider applicability of muon spin spectroscopy be explored. 
Acknowledgments

This work was supported by a travel grant from EPSRC and muon beam time from PSI and ISIS (STFC).

\section{Abbreviations}

TF- $\mu S R$, transverse field muon spin rotation; ALC- $\mu S R$, avoided level crossing muon spin resonance; ESR, electron spin resonance; AOT, bis(2-ethylhexyl) sodium sulphosuccinate (Aerosol OT)

\section{References}

(1) Paul, B.K.; Moulik, S.P. Microemulsions: an overview. J. Dispersion. Sci. Technol. 1997, 18, 301-367.

(2) Zhao, H. Methods for stabilizing and activating enzymes in ionic liquids-a review. J. Chem. Technol. Biotechnol. 2010, 85, 891-907.

(3) Eastoe, J.; B. Warne, B. Nanoparticle synthesis in microemulsions. Curr. Opin. Colloid. Interface Sci. 1996, 1, 80o-805.

(4) Chatenay, D.; Urbach, W.; Cazabat, A.M.; Vacher, M.; Waks, M. Proteins in membrane mimetic systems. Insertion of myelin basic protein into microemulsion droplets. Biophys. J. 1985, 48, 893-898.

(5) Wong, M.; Thomas, J.K; Nowak, T. Structure and state of water in reversed micelles. 3. J. Am. Chem. Soc. 1977, 99, 47304736.

(6) Chowdhary, J.; Ladanyi, B.M. Molecular Simulation Study of Water Mobility in Aerosol-OT Reverse Micelles. J. Phys. Chem. 2011, 115, 6306-6316.

(7) De, T.K.; Maitra, A. Solution behaviour of Aerosol OT in non-polar solvents. Adv. Colloid Interface Sci. 1995, 59, 95-193.

(8) Zana, R. Dynamics of Surfactant Self-Assemblies: Micelles, Microemulsions, Vesicles and Lyotropic Phases. CRC Press: Boca Raton, FL, 2005

(9) Behera, G.B.; Mishra, B.K.; Behera, P.K.; Panda, M. Fluorescent probes for structural and distance effect studies in micelles, reversed micelles and microemulsions. Adv. Colloid Interface Sci. 1999, 82, 1-42.

(10) Almgren, M.; Mays, H. in Handbook of Microemulsion Science and Technology Eds. Kumar, P.; Mittal, K.L. CRC Press 1999 Chapter 19. Time-Resolved Luminescence Quenching in Microemulsions. 605-628.

(11) Howe, A.M.; McDonald, J.A.; Robinson, B.H. Fluorescence quenching as a probe of size domains and critical fluctuations in water-in-oil microemulsions. J. Chem. Soc., Faraday Trans. 1 1987, 83, 1007-1027.

(12) Fletcher, P.D.I.; Howe, A.M.; Robinson, B.H. The Kinetics of Solubilisate Exchange between Water Droplets of a Water-inoil Microemulsion. J. Chem. Soc., Faraday Trans. 1 1987, 83, 9851006.

(13) Yoshioka, H. Exchange of the position of a spin probe in an aerosol OT reversed micelle. J. Colloid Interface Sci., 1983, 95, 8186.

(14) Yoshioka, H. Temperature dependence of motion of a spin probe in aerosol ot reversed micelles. J. Colloid Interface Sci., 1981, 83, 214-220.

(15) Grämpp, G; Rasmussen, K. Nitroxides - Theory, Experiment and Applications Ed. Kokorin, A. I. Chapter 2 Intech 2012 DOI: $10.5772 / 39131$
(16) Yaouanc, A.; deRéotier, P.D. Muon Spin Rotation, Relaxation, and Resonance: Applications to Condensed Matter; International Series of Monographs on Physics, Oxford University Press, Oxford, 2011.

(17) McKenzie, I. The positive muon and $\mu$ SR spectroscopy: powerful tools for investigating the structure and dynamics of free radicals and spin probes in complex systems. Annu. Rep. Prog. Chem., Sect. C: Phys. Chem. 2013, 109, 65-112.

(18) Roduner, E. Polarized positive Muons probing free radicals: a variant of magnetic resonance. Chem. Soc. Rev. 1993, 22, 337-346.

(19) Clayden, N.J. Muons in chemistry. Phys. Scr. 2013, 88, 068507

(20) Scheuermann, R.; Tucker, I.M.; Creeth, A.M.; Dilger, H.; Beck, B.; Roduner, E. Towards the determination of partition coefficients of cosurfactants at surfactant bilayer interfaces by muon spin resonance spectroscopy. Phys. Chem. Chem. Phys., 2002, 4, 1510-1512.

(21) Scheuermann, R.; Tucker, I.M.; Dilger, H.; Staples, E.J.; Ford, G.; Fraser, S.B.; Beck, B.; Roduner, E. Partitioning of small amphiphiles at surfactant bilayer/water interfaces: An avoided level crossing muon spin resonance study. Langmuir 2004, 20, 2652-2659.

(22) Dilger, H.; Martyniak, A.; Scheuermann, R.; Vujosevic, D.; Tucker, I.M.; McKenzie, I.; Roduner, E. An ALC study of spin exchange of a muonated cosurfactant in lamellar phase surfactant dispersions. Phys. B 2006, 374-375, 317-320

(23) Luisi, P.L.; Magid, L.J. Solubilization of enzymes and nucleic acids in hydrocarbon micellar solutions. Crit. Rev. Biochem., 1986, 20, 409-474.

(24) Roduner, E.; Stolmar, M.; Dilger, H.; Reid, I. Reorientation dynamics of cyclohexadienyl radicals in high silica ZSM-5. J. Phys. Chem. 1998, 102, 7591-7597.

(25) Frisch, M.J. et al., Gaussian 03, Revision E.o2; Gaussian, Inc., Wallingford, CT, 2004.

(26) Becke, A.D. Density-functional thermochemistry. III. The role of exact exchange. J. Chem. Phys. 1993, 98, 5648-5652.

(27) Lee, C.; Yang, W.; Parr, R.G. Development of the Colle-Salvetti correlation-energy formula into a functional of the electron density. Phys. Rev. B: Condens. Matter Mater. Phys. 1988, 37, 785789.

(28) Miehlich, B.; Savin, A.; Stoll, H.; Preuss, H. Results obtained with the correlation energy density functionals of becke and Lee, Yang and Parr. Chem. Phys. Lett. 1989, 157, $200-206$.

(29) Krishnan, R.; Binkley, J.S.; Seeger, R.; Pople, J.A. Self-Consistent Molecular Orbital Methods. XX. A Basis Set for Correlated Wavefunctions. J. Chem. Phys. 1980, 72, 650-654.

(30) Barone, V. Structure, Magnetic Properties and Reactivities of Open-Shell Species from Density Functional and Self-Consistent Hybrid Methods. Recent Advances in Density Functional Methods; Part I, Ed. Chong, D.P. World Scientific Publ. Co. 1996.

(31) McKenzie, I.; Cammidge, A.N.; Gopee, H.; Dilger, H.; Scheuermann, R.; Stoykov, A.; Jayasooriya, U.A. Muoniated spin probes in the discotic liquid crystal HHTT: Rapid electron spin relaxation in the hexagonal columnar and isotropic phases. Phys. Rev. E: Stat., Nonlinear, Soft Matter Phys. 2013, 87, 012504.

(32) Yu, D.; Percival, P.W.; Brodovitch, J.-C.; Leung, S.-K.; Kiefl, R.F.; K. Venkateswaran, K.; Cox, S.F.J. Structure and intramolecular motion of muonium-substituted cyclohexadienyl radicals. Chem. Phys. 1990, 142, 229-236.

(33) Tregenna-Piggott, P.L.W.; E. Roduner, E.; Santos, S. Calculation of the avoided level-crossing muon spin resonance response for various stochastic motions using Monte Carlo methods. Chem. Phys. 1996, 203, 317-337. 
(34) Macrae, R.M.; Briere, T.M. The muonium adduct to biacetyl - ab initio calculations and vibrational averaging. Hyperfine Interact. 1997, 106, 169-174.

(35) Yoshino, A.; Okabayashi, H.; Yoshida, T.; Kushida, K. Dynamic Structure and Chirality Effects on ${ }^{1} \mathrm{H}$ and ${ }^{13} \mathrm{C}$ NMR Chemical Shifts for Aerosol OT in Reversed Micelles Assisted by NOESY, ROESY, and ${ }^{13} \mathrm{CT}_{1}$ Studies. J. Phys. Chem. 1996, 100, 9592-9597.

(36) Roduner, E.; Strub, W.; Burkhard, P.; Hochmann, J.; Percival, P.W.; Fischer, H.; Ramos, M.; Webster, B.C. Muonium Substituted Organic Free Radicals in Liquids. Muon-Electron Hyperfine Coupling Constants of Alkyl and Allyl Radicals. Chem. Phys. 1982, $67,275-285$.

(37) Cox, S. F. J.; Sivia, D. S. Longitudinal muon spin relaxation in muonium-like systems. Hyperfine Interact. 1994, 87, 971-976.

(38) Cox, S. F. J. Muon spin relaxation studies of interstitial and molecular motion. Solid State Nucl. Magn. Reson. 1998, 11, 103-121

(39) McKenzie, I.; Dilger, H.; Stoykov, A.; Scheuermann, R. Muon Spin Spectroscopy of the Nematic Liquid Crystal 4-n-Pentyl-4'-cyanobiphenyl (5CB). J. Phys. Chem. B, 2009, 113, 1013510142.

(40) Brigata, G.; Franchi, P.; Lucarini, M.; Pedulli, G.F.; Valgimigli, L. The EPR study of dialkyl nitroxides as probes to investigate the exchange of solutes between micellar and water phases. Res. Chem. Intermed. 2002, 28, 131-141.

(41) Tamura, K.; Schelly, Z.A. Reversed Micelle of Aerosol-OT in Benzene. 3. Dynamicsof the Solubilization of Picric Acid. J. Am. Chem. Soc. 1981, 103, 1018-1022

(42) Harada. S.; Schelly, Z.A. Reversed Micelle of Dodecylpyridinium Iodide in Benzene. Pressure-Jump Relaxation Kinetic and Equilibrium Study of the Solubilization of $7,7,8,8$-Tetracyanoquinodimethane. J. Phys. Chem. 1982, 86, 2098-2102

(43) Alberry, W.J.; Burke, J.F.; Leffler, E.B.; Hagdaft, J. Interfacial Transfer Studied with a Rotating Diffusion Cell. J. Chem. Soc., Faraday Trans. 1 1976, 72, 1618-1626

(44) Fisk, P.R.; Ford, M.G.; Watson, P. in Comprehensive Chemical Kinetics, Eds Compton, R.G; Hancock, G. Elsevier 1999 Volume 37 Applications of Kinetic Modelling Chapter 6 The kinetics of the partitioning of compounds between octanol and water, and its relationship to the movement of molecules in biological systems, 161- 194

(45) Albery, W.J.; Choudhery, R.A.; Atay, N.Z.; Robinson, B.H. Rotating Diffusion Cell Studies of Microemulsion Kinetics. J. Chem. Soc., Faraday Trans. 1 1987, 83, 2407-2419 


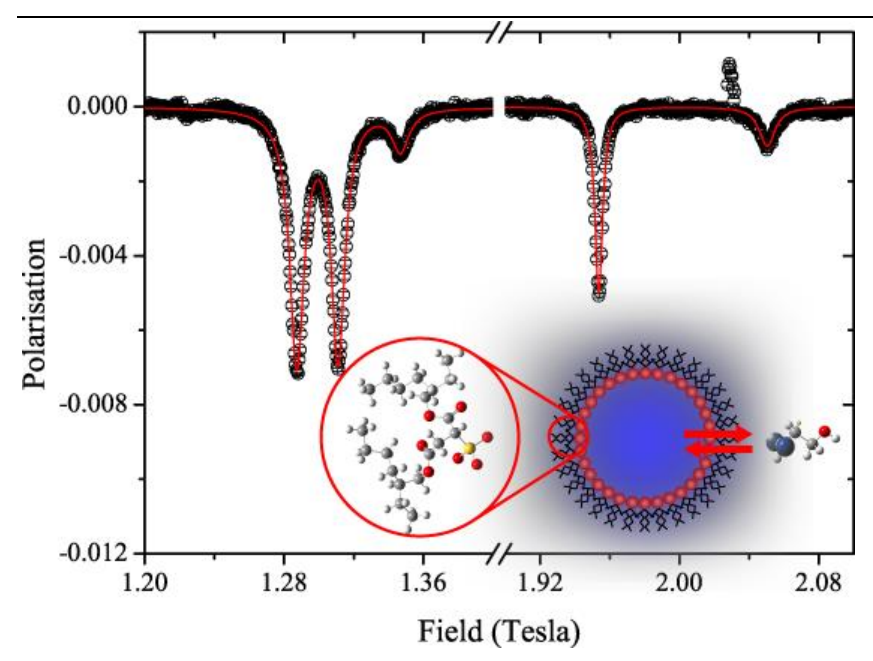

